\title{
Rituximab-induced pneumonitis mimicking miliary tuberculosis
}

\author{
To the Editor:
}

Rituximab, a chimeric anti-CD20 monoclonal antibody, is commonly being used in combination with chemotherapy regimens in the standard treatment of B-cell non-Hodgkin lymphoma (NHL) [1]. Rituximab is considered to be a relatively safe drug but recently, severe and fatal interstitial lung disease (ILD) was noted with an occurrence rate of $0.01-0.03 \%$ [2]. Computed tomography (CT) patterns of rituximabinduced ILD include focal (54\%) or diffused (9\%) alveolar patterns and macronodules (3\%), or groundglass patterns (34\%) [2]. In this regard, we report a patient with NHL in whom ILD developed after six cycles of R-CHOP (rituximab, cyclophosphamide, doxorubicin, vincristine and prednisone) therapy with diffused micronodules mimicking miliary tuberculosis (TB). R-CHOP was discontinued and the patient gradually recovered.

This 54-year-old housewife, who initially presented with abdominal fullness due to intra-abdominal lymphadenopathy, was diagnosed with diffused large B-cell lymphoma. She was treated with R-CHOP three times a week. Several days after the sixth cycle of treatment she reported dyspnoea, fever and cough. Physical examination was unremarkable. Pulse oximetry and analysis of arterial blood gases confirmed the presence of hypoxaemia. A chest radiography and CT scan (fig. 1) showed bilateral diffuse micronodules. Bone marrow biopsy revealed no granuloma or malignancy, and acid-fast stains and culture for mycobacteria were negative. The patient underwent bronchoscopy with bronchoalveolar lavage and transbronchial biopsies. Culture for bacteria, fungi and mycobacteria were all negative. PCR did not detect any Pneumocystis jiroveci DNA. The transbronchial biopsy revealed interstitial fibrosis and type II pneumocyte hyperplasia (fig. 2). No granuloma or malignancy was observed. We prescribed supportive treatment without specific medications (including steroids) and her symptoms improved. 4 months later, CT of the chest also revealed marked improvement and she was asymptomatic.

The common differential diagnosis of randomly distributed micronodules on CT patterns includes miliary $\mathrm{TB}$, miliary fungus and haematogenous metastasis [3]. Because the patient lived in a TB-endemic region and was in an immunocompromised state, we took miliary TB as the first consideration. The final diagnosis of rituximab-induced ILD was mainly made based on the lack of pathological or microbial infection evidence and the dramatic improvement in the patient's condition after the discontinuation of R-CHOP.

The time to onset of rituximab-induced ILD is usually classified as early (day 1), delayed (days 7-21) or late (>30 days). Mechanisms leading to late-onset ILD remain unclear. A potential explanation may be the induction and release of cytokines following rituximab binding to $\mathrm{CD}_{2} 0^{+}$cells $[2,4]$. Because the patient was treated concomitantly with cytotoxic drugs, we recognise that it is difficult to completely rule out cytotoxic

FIGURE 1 Chest computed tomography scan revealing diffused micronodules over bilateral lung field.

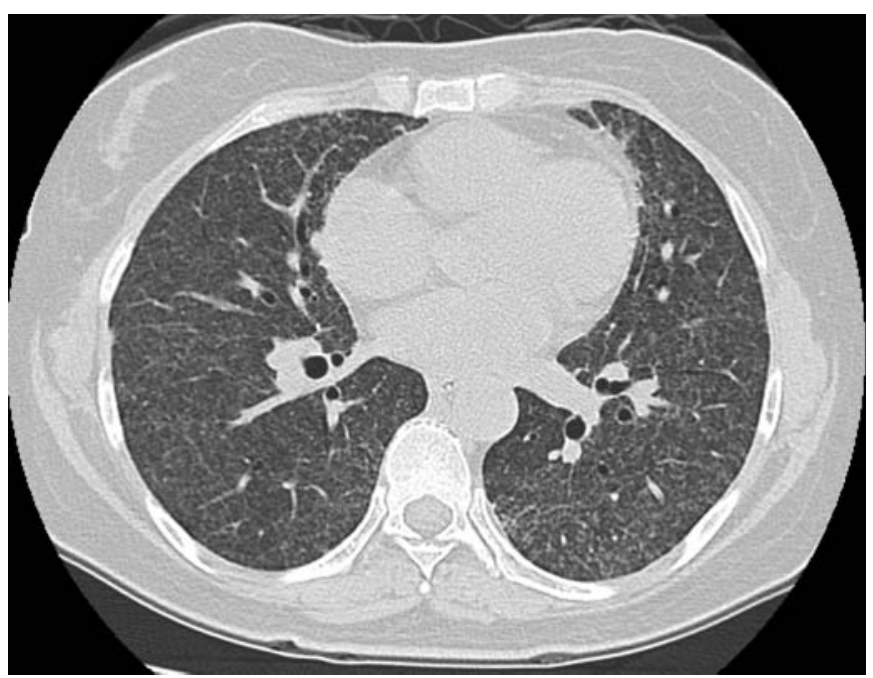




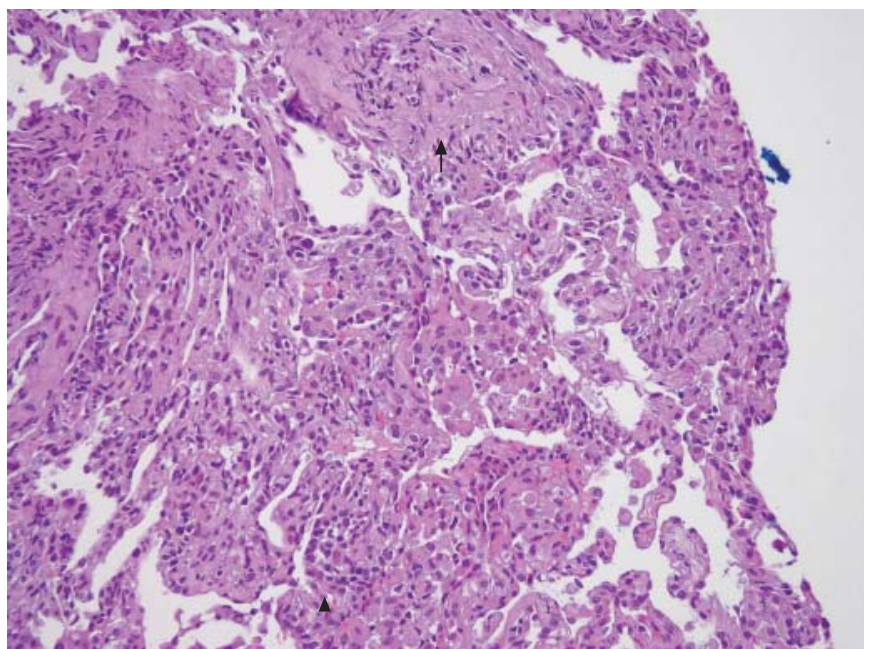

FIGURE 2 The transbronchial biopsy results revealed interstitial fibrosis (black arrow) and type II pneumocyte hyperplasia (arrow head). Haematoxylin and eosin stain. Original magnification $\times 200$.

drug-induced pulmonary toxicity (i.e. cyclophosphamide). However, the incidence is considered to be very low [5].

CT scans of rituximab-induced ILD showed focal alveolar densities in 54\% of patients, in combination with ground-glass attenuation in 32\%. Other presentations include diffused alveolar patterns (9\%) and macronodules $(3 \%)$ [2]. However, ILD with diffused pulmonary micronodules was found in our patient and was never reported. In conclusion, rituximab-induced ILD could present with micronodules mimicking miliary TB and physicians should be aware of this possible complication.

\section{0}

@ERSpublications

Rituximab-induced ILD could present with micronodules mimicking military TB; doctors should be aware of this complication http://ow.ly/o5ETh

Chih-Wei Yao ${ }^{1,2}$, Wei-Chih Liao ${ }^{1,2}$, Chih-Yen Tu ${ }^{1,2,3}$, Hung-Jen Chen ${ }^{1,2,4}$, Guan-Chin Teseng ${ }^{5}$ and Su-Peng Yeh ${ }^{2,6}$

${ }^{1}$ Division of Pulmonary and Critical Care Medicine, China Medical University Hospital, Taichung, ${ }^{2}$ Dept of Internal Medicine, China Medical University Hospital, Taichung, ${ }^{3}$ Dept of Life Sciences, National Chung Hsing University, Taichung, ${ }^{4}$ Dept of Respiratory Therapy, China Medical University, Taichung, ${ }^{5}$ Dept of Pathology, China Medical University Hospital, Taichung, and ${ }^{6}$ Division of Hematology and Oncology, China Medical University Hospital, Taichung, Taiwan.

Correspondence: H-J Chen, Dept of Internal Medicine, China Medical University Hospital, \#2 Yude Road, Taichung 40459, Taiwan. E-mail: redman0127@gmail.com

Received: May 192013 | Accepted after revision: June 052013

Conflict of interest: None declared.

Provenance: Submitted article, peer reviewed.

\section{References}

1 Coiffier B, Lepage E, Briere J, et al. CHOP chemotherapy plus rituximab compared with CHOP alone in elderly patients with diffuse large-B-cell lymphoma. N Engl J Med 2002; 346: 235-242.

2 Liote H, Liote F, Seroussi B, et al. Rituximab-induced lung disease: a systematic literature review. Eur Respir J 2010; 35: 681-687.

3 High-resolution computed tomography findings of lung disease. In: Webb WR, Müller NL, Naidich DP, eds. HighResolution CT of the Lung. Philadelphia, Lippincott Williams \& Wilkins, 2009; p. 113.

4 Bienvenu J, Chvetzoff R, Salles G, et al. Tumor necrosis factor alpha release is a major biological event associated with rituximab treatment. Hematol J 2001; 2: 378-384.

5 Herishanu Y, Polliack A, Leider-Trejo L, et al. Fatal interstitial pneumonitis related to rituximab-containing regimen. Clin Lymphoma Myeloma 2006; 6: 407-409.

Eur Respir Rev 2013; 22: 587-588 | DOI: 10.1183/09059180.00003813 | Copyright @eERS 2013 ERR articles are open access and distributed under the terms of the Creative Commons Attribution Non-Commercial Licence 3.0. 